\title{
EDITOR'S
}

\section{How Dangerous is Testosterone Supplementation?}

Recently, significant media attention has been focused on testosterone supplementation therapy (TST) and increased cardiovascular (CV) risk. Treatment for testosterone deficiency syndrome (TDS) has been on the rise in the past several years. US Food \& Drug Administration (FDA) drug utilization data showed sales increased 65\% between 2009 and 2013 (1). The true prevalence of TDS varies based on the source, definition of the condition and age of population studied, however, it is believed to range from 2-39\% $(2,3)$. Patients suffering from TDS are at an irrefutably increased risk for bone density loss, development of type 2 diabetes (T2DM), anemia, sarcopenia, cognitive deficits, and premature death (4). Shores et al, have shown increased mortality rates in men with low testosterone (mortality rates with normal, equivocal, and low testosterone levels were 20\%, 25\%, 35\% respectively) (5).

Patients should be made aware of possible adverse events associated with TST in particular as polycythemia and hyperestrogenism (6). Polycythemia is believed to be a dose-dependent effect and hematocrit levels should be maintained below 54\% to prevent hyperviscosity-related events. Hyperestrogenism is a response of testosterone conversion to estrogen via aromatase in adipose tissue and may be associated with gynecomastia. Two recent meta-analyses have shown that prostate related events (rates of prostate cancer, PSA $>4 \mathrm{ng} / \mathrm{mL}$, prostate biopsies) were not statistically significantly higher in the testosterone supplementation group compared to the placebo group $(6,7)$. Also these meta-analyses demonstrated no significant elevation in CV risk. In a meta-analysis by Aruajo et al., the authors reported that testosterone levels $\geq 2.18$ standard deviations below normal range were associated with 35\% and 25\% elevated risk in all-cause mortality and CV disease mortality, respectively (8). However, the authors note that their analysis showed considerable between-study heterogeneity limiting the clinical extrapolation of the data.

Some of the difficulty addressing research in TST relates to the methodological challenges related to studying patients with TDS, including: population heterogeneity, circadian rhythm of testosterone secretion, use of total vs. free testosterone, number of pre-treatment testosterone levels, covariate analysis, duration and follow-up of TST. There is often significant heterogeneity in the populations being studied therefore limiting the validity of comparative analyses. Ideally, populations should be matched in patient demographics, and baseline TDS parameters (symptoms and severity). Trial design should account for testosterone level variance based on circadian rhythm and consistently use total (by LCMS analysis) or free testosterone (by equilibrium dialysis) level measurement. Also, important for trial design are standardized minimum number of pre-treatment testosterone levels, standardized duration of treatment and follow-up 


\section{EDITOR'S}

protocols. Statistical analyses should be appropriate (routinely used and interpretable by the average scientific reader) and validated for the type of study.

There have been three articles popularized by the media in the last couple years. Basaria et al. published in the New England Journal of Medicine a randomized, double-blinded, placebo-controlled trial in men with TDS (total testosterone [TT]100-350 ng/ dL) older than 65 years of age with the primary end-point of assessing fraility (mobility) data (in particular quadriceps strength) (9). Patients were treated for 6 months (T gel vs. placebo) and had TT levels maintained between 500-1000 ng/dL. Once again, neither primary nor secondary endpoints were CV events. The CV and atherosclerotic events were recorded via self-report or medical record review or physician assessment. In all, 209 patients were randomized and 176 completed the 6 month treatment period. of note, there was a higher rate of prior CV morbidities in the T supplementation group at baseline. The authors reported more CV events in the testosterone group compared to placebo ( 23 vs. 5 subjects), indeed the study was ceased prematurely because of this signal. However, some of the symptoms defined as CV in nature would not put a patient at increased risk of significant morbidity or mortality (peripheral edema, systolic hypertension, atrial tachyarrythmias). It is unclear whether any significant difference would have been demonstrated between the groups if myocardial infarction (MI) and cerebrovascular accident (CVA) had been used to define CV events.

In another such article, Vigen et al. published in JAMA a retrospective review of men with low TT $(<300 \mathrm{ng} / \mathrm{dL})$ who underwent coronary angiography (CA) in the Veterans Affairs (VA) hospital system (10). Men who were on TST prior to CA were excluded from the study. Only the TT level closest to the CA was used. It was assumed that patients adhered to TST the entire study period based on filling an initial prescription from the VA pharmacy. Primary end-points were time to all-cause mortality and time to hospitalization for MI or CVA. They used Cox proportional hazards models with a rarely utilized statistical test known as 'stabilized inverse probability of treatment weighting' to control for over 50 different variables to assess the relationship between TST and the primary endpoints. There was no control used for time of day in TT testing. There were 8709 patients included in the study and of these 1223 (14\%) initiated TST a median of 531 days after the angiography. However, after publication the authors adjusted their reported data and changed the number of patients excluded due to starting TST after MI or stroke from 128 to 1132, all of whom, we believe, should have been included in the non-TST group within the study. Only 60\% of TST patients had a follow-up TT level checked while on therapy and 18\% of the TST group only filled one prescription. The outcomes were as follows: death - non-TST 9\%, TST 12\%; MI - non-TST 6\%, TST 2\%; CVA - non-TST 6\%, TST 3\%. Yet, through obtuse statistical analysis weighting for 50 confounders, many of which are not classically associated with TDS, the authors suggested CV event were three times more likely in the TST group. The major limitations to this study included: convoluted and complex statistical analysis, data errors within the original paper, erroneous exclusion criteria, flawed laboratory testing (time not factored in TT), inadequate documentation of TST, insufficient follow-up of TST patients. 


\section{EDITOR'S}

More recently, Finkle et al. published in PLoS ONE a retrospective cohort study using an insurance claims registry database. The study was conducted by a private firm in conjunction with the NIH. They used the insurance database to access diagnosis, prescriptions, and procedures but they had no data on TT levels or indications for treatment. The study group included patients who filled a prescription for testosterone $(n=55,593)$ and the control group were patients who filled a prescription for a phosphodiesterase type-5 inhibitor (PDE5i) ( $\mathrm{n}=167,279)$. Patients were followed for 90 days post-prescription and covariates were determined from data drawn from 12 months pre-prescription. They had no data on medication use compliance. The primary endpoint was non-fatal MI within 90 days of filling the prescription. They analyzed MI rates pre and post testosterone prescription. They found that men on TST over 65 years of age (RR=2.19 vs. 1.15) and men under 65 years with prior history of heart disease ( $R R=2.9$ vs. 1.4 ) had higher relative risks (RR) of non-fatal MI when compared to their PDE5i counterparts. The major limitations of this study included: the fact that all data were based on prescription data, questionable control group, short follow-up (90 days), and questionable conflict of interest with authors (one of the authors was owner of the private group analyzing the data). It is being increasingly appreciated that PDE5i may be cardioprotective, thus using them as a control group in a study assessing MI events is flawed and essentially invalidates the data (11).

Overall, these three papers claiming increased CV risks in patient using TST are all inherently flawed and limit the interpretation of the data and the clinical applicability is questionable. Especially when more than two decades of good science and the Araujo meta-analysis have found quite the opposite, that TDS is associated with a higher incidence of CV events. Finally, more recently, Corona et al show in a large meta-analysis no association between TST and increased CV risk (12). In September 2014, a US Food and Drug Administration advisory panel met and determined more studies are needed to identify the true CV risk with TST and they recommended changing labeling to stress use only in those patients with a diagnosis of hypogonadism (1). In December 2014, the European Medicines Agency's Pharmacovigilance Risk Assessment Committee determined there was no consistent evidence of an increased CV risk with the use of TST (13). With this, the media attention surrounding TST has declined and most importantly, our clinical practice regarding TST prescribing and what we tell patients has not changed.

\section{REFERENCES}

1. Tucker ME. FDA Advisory Panel Urges Restrictions on Testosterone Use. In. Medscape; 2014; in press.

2. Mulligan T, Frick MF, Zuraw QC, Stemhagen A, McWhirter C. Prevalence of hypogonadism in males aged at least 45 years: the HIM study. Int J Clin Pract.2006;60:762-9.
3. Allan CA, McLachlan RI. Age-related changes in testosterone and the role of replacement therapy in older men. Clin Endocrinol (0xf). 2004;60:653-70.

4. Traish AM, Miner MM, Morgentaler A, Zitzmann M. Testosterone deficiency. Am J Med. 2011;124:578-87. 


\section{EDITOR'S

5. Shores MM, Matsumoto AM, Sloan KL, Kivlahan DR. Low serum testosterone and mortality in male veterans. Arch Intern Med. 2006;166:1660-5.

6. Calof $\mathrm{OM}$, Singh $\mathrm{AB}$, Lee ML, Kenny AM, Urban RJ, Tenover JL, et al. Adverse events associated with testosterone replacement in middle-aged and older men: a meta-analysis of randomized, placebocontrolled trials. J Gerontol A Biol Sci Med Sci. 2005;60:1451-7.

7. Fernández-Balsells MM, Murad MH, Lane $M$, Lampropulos JF, Albuquerque F, Mullan RJ, et al. Clinical review 1: Adverse effects of testosterone therapy in adult men: a systematic review and metaanalysis. J Clin Endocrinol Metab. 2010;95:2560-75.

8. Araujo AB, Dixon JM, Suarez EA, Murad MH, Guey LT, Wittert GA. Clinical review: Endogenous testosterone and mortality in men: a systematic review and metaanalysis. J Clin Endocrinol Metab. 2011;96:3007-19.

9. Basaria $S$, Coviello AD, Travison TG, Storer TW, Farwell WR, Jette AM,et al. Adverse events associated with testosterone administration. N Engl J Med. 2010;363:109-22.

10. Vigen R, O'Donnell $\mathrm{Cl}$, Barón AE, Grunwald GK, Maddox TM, Bradley SM,et al. Association of testosterone therapy with mortality, myocardial infarction, and stroke in men with low testosterone levels. JAMA. 2013;310:1829-36.
11. Kukreja RC. Sildenafil and cardioprotection. Curr Pharm Des. 2013;19:6842-7

12. Corona G, Maseroli E, Rastrelli G, Isidori AM, Sforza A, Mannucci E, et al.Cardiovascular risk associated with testosterone-boosting medications: a systematic review and meta-analysis. Expert Opin Drug Saf. 2014;13:1327-51.

13. No consistent evidence of an increased risk of heart problems with testosterone medicines. In: European Medicines Agency Nov 21 2014. Available at: http:// www.ema.europa.eu/ema/index.jsp?curl=pages/ news_and_events/news/2014/11/news_ detail_002218.jsp\&mid=WC0b01ac058004d5c1

Lawrence C. Jenkins, MD, PhD and John P. Mulhall, MD, PhD

Director, Sexual \& Reproductive Medicine Urology Service

Memorial Sloan-Kettering Cancer Center 1275 York Ave

New York, New York 10065, USA Fax: +1 646 888-6452

E-mail: mulhalj1@mskcc.org 